G. SCHOBER

KODAI MATH. J.

2 (1979), 411-419

\title{
COEFFICIENTS OF INVERSES OF UNIVALENT FUNCTIONS WITH QUASICONFORMAL EXTENSIONS
}

\author{
By GLenN Schober
}

\section{$\S 1$. Introduction.}

Let $\Sigma^{\prime}$ denote the family of univalent functions

$$
g(z)=z+\sum_{n=1}^{\infty} \frac{b_{n}}{z^{n}}
$$

in $\tilde{\Delta}=\{z: 1<|z|<\infty\}$. For $0 \leqq k<1$ let $\Sigma_{k}^{\prime}$ be the family of functions in $\Sigma^{\prime}$ that admit $k$-quasiconformal extensions to $\bar{J}=\{z:|z| \leqq 1\}$. That is, each $g \in \Sigma_{k}^{\prime}$ has a homeomorphic extension to $\bar{J}$, that is absolutely continuous on a. e. horizontal and vertical line in $\bar{\Delta}$ and satisfies

$$
\left|g_{\bar{z}}\right| \leqq k\left|g_{z}\right| \quad \text { a. e. in } \bar{\Delta} \text {. }
$$

If $k=0$, then $g$ is an entire univalent function. Consequently, $\Sigma_{0}^{\prime}$ contains only the identity function. As $k \rightarrow 1$, the families $\Sigma_{k}^{\prime}$ are dense in $\Sigma^{\prime}$, and we therefore define $\Sigma_{1}^{\prime}=\Sigma^{\prime}$. Since $\Sigma_{k_{1}}^{\prime} \subset \Sigma_{k_{2}}^{\prime}$ for $k_{1}<k_{2}$, the families $\Sigma_{k}^{\prime}$ interpolate in a monotonic fashion from the identity function to the family $\Sigma^{\prime}$.

R. Kühnau [2] and O. Lehto [5] have obtained the sharp coefficient estimates

$$
\left|b_{1}\right| \leqq k \text { and }\left|b_{2}\right| \leqq(2 / 3) k
$$

for functions $g \in \sum_{k}^{\prime}$. In this article we shall study the coefficients of their inverse functions.

That is, if $G$ is the inverse of a function $g$ in $\Sigma_{k}^{\prime}$, i. e., $G=g^{-1}$, then $G$ has an expansion

$$
G(w)=w+\sum_{n=1}^{\infty} \frac{B_{n}}{w^{n}}
$$

in some neighborhood of $w=\infty$. Since $B_{1}=-b_{1}$ and $B_{2}=-b_{2}$, the sharp estimates

$$
\left|B_{1}\right| \leqq k \quad \text { and } \quad\left|B_{2}\right| \leqq(2 / 3) k
$$

This work was supported in part by a grant from the National Science Foundation. Received July 26, 1978 
are a consequence of (1). that

For the class $\Sigma_{1}^{\prime}=\Sigma^{\prime}, G$. Springer [8] proved that $\left|B_{3}\right| \leqq 1$ and conjectured

$$
\left|B_{2 n-1}\right| \leqq \frac{(2 n-2) !}{n !(n-1) !}, \quad n=3,4,5, \cdots .
$$

Very recently, Y. Kubota [1] and the present author [7] have verified (3) for $2 \leqq n \leqq 7$. Based on a technique of Lehto [5] we may therefore conclude that

$$
\left|B_{2 n-1}\right| \leqq \frac{(2 n-2) !}{n !(n-1) !} k
$$

for $g \in \Sigma_{k}^{\prime}$ and $2 \leqq n \leqq 7$.

It is the purpose of this article to improve the estimates (4). We shall also obtain anestimate when $n=8$ and verify the conjecture (3) for $n=8$ as a special case.

\section{$\S 2$. Results.}

The following theorem contains the results of this article. Its proof will be given in Section 5 .

PRINCIPAL TheOREM. Let $g$ belong to $\Sigma_{k}^{\prime}, 0 \leqq k \leqq 1$, and let

$$
G(w)=g^{-1}(w)=w+\sum_{n=1}^{\infty} \frac{B_{n}}{w^{n}}
$$

be the expansion of its inverse function in a neighborhood of $w=\infty$. Then

$$
\begin{aligned}
& \left|B_{3}\right| \leqq k-\frac{1}{2} k(1-k) \leqq k \\
& \left|B_{5}\right| \leqq 2 k-\frac{1}{3 !} k(1-k)(10+7 k) \leqq 2 k \\
& \left|B_{7}\right| \leqq 5 k-\frac{1}{4 !} k(1-k)\left(114+103 k+49 k^{2}\right) \leqq 5 k \\
& \left|B_{9}\right| \leqq 14 k-\frac{1}{5 !} k(1-k)\left(1656+1606 k+1181 k^{2}+451 k^{3}\right) \leqq 14 k \\
& \left|B_{11}\right| \leqq 42 k-\frac{1}{6 !} k(1-k)\left(30120+29846 k+26381 k^{2}+17776 k^{3}+6241 k^{4}\right) \leqq 42 k \\
& \left|B_{13}\right| \leqq 132 k-\frac{1}{7 !} k(1-k)\left(664560+662796 k+631632 k^{2}+529887 k^{3}\right. \\
& \left.\quad+317892 k^{4}+98841 k^{5}\right) \leqq 132 k \\
& \left|B_{15}\right| \leqq 429 k-\frac{3}{8 !} k(1-k)\left(5764080+5759724 k+5658280 k^{2}+5247149 k^{3}\right.
\end{aligned}
$$


Since $\Sigma_{0}^{\prime}$ contains only the identity function, the theorem is trivial in this case. The case $k=1$ is of special interest:

COROLLARY. If $g$ belongs to $\Sigma^{\prime}$, then the coefficients of its inverse function satisfy

$$
\begin{aligned}
& \left|B_{3}\right| \leqq 1, \quad\left|B_{5}\right| \leqq 2, \quad\left|B_{7}\right| \leqq 5, \quad\left|B_{9}\right| \leqq 14 \\
& \left|B_{11}\right| \leqq 42, \quad\left|B_{13}\right| \leqq 132, \quad \text { and } \quad\left|B_{15}\right| \leqq 429
\end{aligned}
$$

Equality in any of these occurs if and only if $g(z)=z+\frac{e^{2 \alpha}}{z}$ for some real $\alpha$.

In this special case the result for $\left|B_{15}\right|$ is new. One easily verifies that equality occurs (also in (3)) for the indicated functions. To see that these are the only extremal functions, we observe at the end of Section 5 that equality can occur only if $\left|b_{1}\right|=1$, which by the Area Theorem (see Section 3) can occur only for the indicated functions. For $0<k<1$ we do not assert that the estimates of the Theorem are sharp.

\section{$\S 3$. The Principal Lemma.}

The set $H(\tilde{\Delta})$ of all analytic functions on $\tilde{\Delta}$ with the topology of locally uniform convergence is a locally convex topological vector space. We denote its topological dual space by $H^{\prime}(\tilde{\Delta})$. If $h(z, \zeta)$ is analytic in $\tilde{\Delta} \times \tilde{\Delta}$ and $L \in H^{\prime}(\tilde{\Delta})$, we define

$$
L^{2}(h(z, \zeta))=L(L(h(z, \zeta))) \quad \text { and } \quad|L|^{2}(h(z, \zeta))=L(\overline{L(h(z, \bar{\zeta}))})
$$

where $L$ is applied first to the function of $z$ and then to the function of $\zeta$. In this framework we state (cf. [3; 6, Theorem 14.15]):

Grunsky-Kühnau Inequalitıes. If $g \in \Sigma_{k}^{\prime}, 0 \leqq k \leqq 1$, and $L \in H^{\prime}(\tilde{\Delta})$, then

$$
\left|L^{2}\left(\log \frac{g(z)-g(\zeta)}{z-\zeta}\right)\right| \leqq k|L|^{2}\left(\log \frac{1}{1-(z \bar{\zeta})^{-1}}\right)
$$

These inequalities may be "exponentiated" in the following manner. If $\phi(w)$ $=\sum_{n=0}^{\infty} c_{n} w^{n}$ is any entire function and $\phi^{+}(w)=\sum_{n=0}^{\infty}\left|c_{n}\right| w^{n}$, then [6, Theorem 11.16]

$$
\left|L^{2}\left(\phi \circ \log \frac{g(z)-g(\zeta)}{z-\zeta}\right)\right| \leqq|L|^{2}\left(\phi^{+} \circ \log \left[1-(z \bar{\zeta})^{-1}\right]^{-k}\right) .
$$

In particular, if $\phi(w)=e^{-w}$, then

$$
\begin{aligned}
& \left|L^{2}\left(\frac{z-\zeta}{g(z)-g(\zeta)}\right)\right| \leqq|L|^{2}\left(\left[1-(z \bar{\zeta})^{-1}\right]^{-k}\right) \\
& \quad=\sum_{m=0}^{\infty} \frac{k(k+1) \cdots(k+m-1)}{m !}\left|L\left(z^{-m}\right)\right|^{2} .
\end{aligned}
$$


We now distinguish a special functional $L$. Fix $g \in \Sigma_{k}^{\prime}$ and $n \geqq 1$. Denote $G(w)=g^{-1}(w)=w+\sum_{\nu=1}^{\infty} \frac{B_{\nu}}{w^{\nu}}$, and define $L$ to be the functional that associates to $h \in H(\tilde{J})$ the coefficient $d_{n}$ in the expansion of $h \circ G(w)=\sum_{\nu=-\infty}^{\infty} \frac{d_{\nu}}{w^{\nu}}$ in a neighborhood of $w=\infty$. Then

$$
L^{2}\left(\frac{z-\zeta}{g(z)-g(\zeta)}\right)=B_{2 n-1}
$$

and we have the following consequence of (5).

PRINCIPAL LEMmA. If $g \in \Sigma_{k}^{\prime}, 0 \leqq k \leqq 1$, and $G(w)=g^{-1}(w)=w+\sum_{\nu=1}^{\infty} \frac{B_{\nu}}{w^{\nu}}$ in $a$ nerghborhood of $w=\infty$, then

$$
\left|B_{2 n-1}\right| \leqq \frac{k(k+1) \cdots(k+n-1)}{n !}+\sum_{m=1}^{n-2} \frac{k(k+1) \cdots(k+m-1)}{m !}\left|B_{n}^{(-m)}\right|^{2}
$$

for $n \geqq 1$, where $[G(w)]^{-m}=\frac{1}{w^{m}}+\sum_{\nu=m+2}^{\infty} \frac{B_{\nu}^{(-m)}}{w^{\nu}}$ in a neighborhood of $w=\infty$. The sum in (6) is omitted for $n=1$ and $n=2$.

The inequality (6) is our main tool. We shall also use the following Area Theorem of Kühnau [3] and Lehto [4].

AREA Theorem. If $g(z)=z+\sum_{n=1}^{\infty} \frac{b_{n}}{z^{n}}$ belongs to $\sum_{k}^{\prime}, 0 \leqq k \leqq 1$, then $\sum_{n=1}^{\infty} n\left|b_{n}\right|^{2}$ $\leqq k^{2}$. In partıcular, $\left|b_{1}\right| \leqq k$ with equality if and only if $g(z)=z+\frac{e^{\imath \alpha} k}{z}$ for some real $\alpha$.

The Area Theorem will be used in the following form. then

COROLlARY. If $g(z)=z+\sum_{n=1}^{\infty} \frac{b_{n}}{z^{n}}$ belongs to $\Sigma_{k}^{\prime}, 0 \leqq k \leqq 1$, and $r, s$, $t$ are real,

$$
\left(r\left|b_{6}\right|+s\left|b_{4}\right|+t\left|b_{2}\right|\right)^{2} \leqq\left(\frac{r^{2}}{6}+\frac{s^{2}}{4}+\frac{t^{2}}{2}\right)\left(k^{2}-\left|b_{1}\right|^{2}\right)
$$

Proof. We have

$$
\begin{aligned}
0 & \leqq \frac{2}{3}\left(r\left|b_{4}\right|-\frac{3}{2} s\left|b_{6}\right|\right)^{2}+\frac{1}{3}\left(r\left|b_{2}\right|-3 t\left|b_{6}\right|\right)^{2}+\frac{1}{2}\left(s\left|b_{2}\right|-2 t\left|b_{4}\right|\right)^{2} \\
& =\left(\frac{r^{2}}{6}+\frac{s^{2}}{4}+\frac{t^{2}}{2}\right)\left(6\left|b_{6}\right|^{2}+4\left|b_{4}\right|^{2}+2\left|b_{2}\right|^{2}\right)-\left(r\left|b_{6}\right|+s\left|b_{4}\right|+t\left|b_{2}\right|\right)^{2} \\
& \leqq\left(\frac{r^{2}}{6}+\frac{s^{2}}{4}+\frac{t^{2}}{2}\right)\left(k^{2}-\left|b_{1}\right|^{2}\right)-\left(r\left|b_{6}\right|+s\left|b_{4}\right|+t\left|b_{2}\right|\right)^{2}
\end{aligned}
$$


with the help of the Area Theorem.

\section{§4. Coefficient Relations.}

Several coefficient relations will be needed. If $g(z)=z+\sum_{n=1}^{\infty} \frac{b_{n}}{z^{n}}$ and $G(w)=$ $g^{-1}(w)=w+\sum_{n=1}^{\infty} \frac{B_{n}}{w^{n}}$ in a neighborhood of $\infty$, then

$$
\begin{array}{ll}
B_{1}=-b_{1} & B_{4}=-b_{4}-3 b_{1} b_{2} \\
B_{2}=-b_{2} & B_{5}=-b_{5}-4 b_{1} b_{3}-2 b_{2}^{2}-2 b_{1}^{3} \\
B_{3}=-b_{3}-b_{1}^{2} & B_{6}=-b_{6}-5 b_{1} b_{4}-5 b_{2} b_{3}-10 b_{1}^{2} b_{2} .
\end{array}
$$

Furthermore, if $[G(w)]^{-m}=\frac{1}{w^{m}}+\sum_{\nu=m+2}^{\infty} \frac{B_{\nu}^{(-m)}}{w^{\nu}}$ in a neighborhood of $w=\infty$, for $m \geqq 1$, then

$$
\begin{array}{ll}
B_{3}^{(-1)}=-B_{1} & B_{5}^{(-3)}=-3 B_{1} \\
B_{4}^{(-1)}=-B_{2} & B_{6}^{(-3)}=-3 B_{2} \\
B_{5}^{(-1)}=-B_{3}+B_{1}^{2} & B_{7}^{(-3)}=-3 B_{3}+6 B_{1}^{2} \\
B_{6}^{(-1)}=-B_{4}+2 B_{1} B_{2} & B_{8}^{(-3)}=-3 B_{4}+12 B_{1} B_{2} \\
B_{7}^{(-1)}=-B_{5}+2 B_{1} B_{3}+B_{2}^{2}-B_{1}^{3} & \\
B_{8}^{(-1)}=-B_{6}+2 B_{1} B_{4}+2 B_{2} B_{3}-3 B_{1}^{2} B_{2} & B_{6}^{(-4)}=-4 B_{1} \\
B_{4}^{(-2)}=-2 B_{1} & B_{7}^{(-4)}=-4 B_{2} \\
B_{5}^{(-2)}=-2 B_{2} & B_{8}^{(-4)}=-4 B_{3}+10 B_{1}^{2} \\
B_{6}^{(-2)}=-2 B_{3}+3 B_{1}^{2} & B_{7}^{(-5)}=-5 B_{1} \\
B_{7}^{(-2)}=-2 B_{4}+6 B_{1} B_{2} & B_{8}^{(-5)}=-5 B_{2} \\
B_{8}^{(-2)}=-2 B_{5}+6 B_{1} B_{3}+3 B_{2}^{2}-4 B_{1}^{3} & B_{8}^{(-6)}=-6 B_{1}
\end{array}
$$

\section{$\S 5$. Estimates.}

The Principal Lemma will be applied for $1 \leqq n \leqq 8$. It will be convenient to set $\beta=\left|b_{1}\right|=\left|B_{1}\right|$. Then $0 \leqq \beta \leqq k$ for the family $\Sigma_{k}^{\prime}$ by the Area Theorem.

$n=1$. In this case (6) is identical to the first half of (2).

$n=2$. In this case (6) gives $\left|B_{3}\right| \leqq 1 / 2 k(k+1)=k-1 / 2 k(1-k)$ directly.

$n=3$. Since $\left|B_{3}^{(-1)}\right|^{2}=\beta^{2} \leqq k^{2}$, the estimate (6) leads to 


$$
\left|B_{5}\right| \leqq \frac{k(k+1)(k+2)}{3 !}+k^{3}=2 k-\frac{1}{3 !} k(1-k)(10+7 k) .
$$

$n=4$. We use $\left|B_{4}^{(-2)}\right|^{2}=4 \beta^{2}$ and apply (7) to $\left|B_{4}^{(-1)}\right|^{2}=\left|b_{2}\right|^{2} \leqq(1 / 2)\left(k^{2}-\beta^{2}\right)$. With these relations the inequality (6) reduces to

$$
\left|B_{7}\right| \leqq \frac{k}{4 !}\left[\left(6+11 k+18 k^{2}+k^{3}\right)+12(3+4 k) \beta^{2}\right] .
$$

Since the coefficient of $\beta^{2}$ is positive, we may estimate $\beta^{2}$ by $k^{2}$ and rewrite the resulting bound in the form stated in the Principal Theorem.

$n=5$. We substitute the bounds

$$
\begin{aligned}
& \left|B_{5}^{(-3)}\right|^{2}=9 \beta^{2}, \quad\left|B_{5}^{(-2)}\right|^{2}=4\left|b_{2}\right|^{2} \leqq 2\left(k^{2}-\beta^{2}\right), \\
& \left|B_{5}^{(-1)}\right|^{2} \leqq\left[\left|B_{3}\right|+\beta^{2}\right]^{2} \leqq\left[(1 / 2) k(k+1)+\beta^{2}\right]^{2}
\end{aligned}
$$

into (6) to obtain

$$
\left|B_{9}\right| \leqq \frac{k}{5 !}\left[\left(24+50 k+185 k^{2}+190 k^{3}+31 k^{4}\right)+60\left(4+9 k+5 k^{2}\right) \beta^{2}+120 \beta^{4}\right] .
$$

Since the coefficients of $\beta^{2}$ and $\beta^{4}$ are positive, we may replace $\beta$ by $k$ and rewrite the resulting bound in the form of the Principal Theorem.

$n=6$. Making use of (7), we substitute the bounds

$$
\begin{aligned}
& \left|B_{6}^{(-4)}\right|^{2}=16 \beta^{2}, \quad\left|B_{6}^{(-3)}\right|^{2}=9\left|b_{2}\right|^{2} \leqq \frac{9}{2}\left(k^{2}-\beta^{2}\right), \\
& \left|B_{6}^{(-2)}\right|^{2} \leqq\left[2\left|B_{3}\right|+3 \beta^{2}\right]^{2} \leqq\left[k(k+1)+3 \beta^{2}\right]^{2}, \\
& \left|B_{6}^{(-1)}\right|^{2}=\left|b_{4}+5 b_{1} b_{2}\right|^{2} \leqq\left(\frac{1}{4}+\frac{25}{2} \beta^{2}\right)\left(k^{2}-\beta^{2}\right)
\end{aligned}
$$

into (6) and obtain the estimate

$$
\begin{aligned}
\left|B_{11}\right| \leqq \frac{k}{6 !}[( & \left.120+274 k+1845 k^{2}+2785 k^{3}+1635 k^{4}+361 k^{5}\right) \\
& \left.+60\left(27+97 k+261 k^{2}+44 k^{3}\right) \beta^{2}-360(16-9 k) \beta^{4}\right] .
\end{aligned}
$$

Since the coefficient of $\beta^{4}$ is negative, the estimate

$$
-\beta^{4} \leqq k^{4}-2 k^{2} \beta^{2}
$$

leads to

$$
\begin{gathered}
\left|B_{11}\right| \leqq \frac{k}{6 !}\left[\left(120+274 k+1845 k^{2}+2785 k^{3}+7395 k^{4}-2879 k^{5}\right)\right. \\
\left.+60\left(27+97 k+69 k^{2}+152 k^{3}\right) \beta^{2}\right] .
\end{gathered}
$$

The coefficient of $\beta^{2}$ is positive, and so we may estimate $\beta^{2}$ by $k^{2}$ and rearrange the result into the form given in the Principal Theorem. 
$n=7$. In this case we use (7), the bounds already obtained for $\left|B_{3}\right|$ and $\left|B_{5}\right|$, and the estimates $\beta \leqq k, \beta^{3} \leqq k \beta^{2}, \beta^{6} \leqq k^{2} \beta^{4}$ to arrive at

$$
\begin{aligned}
\left|B_{7}^{(-5)}\right|^{2}= & 25 \beta^{2}, \quad\left|B_{7}^{(-4)}\right|^{2}=16\left|b_{2}\right|^{2} \leqq 8\left(k^{2}-\beta^{2}\right), \\
\left|B_{7}^{(-3)}\right|^{2} \leqq & {\left[3\left|B_{3}\right|+6 \beta^{2}\right]^{2} \leqq\left[\frac{3}{2} k(k+1)+6 \beta^{2}\right]^{2}, } \\
\left|B_{7}^{(-2)}\right|^{2}= & \left|2 b_{4}+12 b_{1} b_{2}\right|^{2} \leqq \\
\left|B_{7}^{(-1)}\right|^{2} \leqq & {\left.\left[\left|B_{5}\right|+2\left|B_{3}\right| \beta+\left|b_{2}\right|^{2}+\beta^{3}\right]^{2}\right)\left(k^{2}-\beta^{2}\right), } \\
\leqq & \left(\left|B_{5}\right|^{2}+4 k\left|B_{3}\right|\left|B_{5}\right|\right)+\left(4\left|B_{3}\right|^{2}+2 k\left|B_{5}\right|\right) \beta^{2}+\left(4\left|B_{3}\right|+k^{2}\right) \beta^{4} \\
& +\left(\left|B_{5}\right|+2 k\left|B_{3}\right|+k \beta^{2}\right)\left(k^{2}-\beta^{2}\right)+\frac{1}{4}\left(k^{2}-\beta^{2}\right)^{2} \\
\leqq & \frac{1}{36}\left(4 k^{2}+48 k^{3}+160 k^{4}+240 k^{5}+133 k^{6}\right)+\frac{1}{6}\left(-2 k-2 k^{2}+11 k^{3}+20 k^{4}\right) \beta^{2} \\
& +\frac{1}{4}\left(1+4 k+12 k^{2}\right) \beta^{4} .
\end{aligned}
$$

Substitution of these bounds into (6) leads to

$$
\begin{aligned}
\left|B_{13}\right| \leqq \frac{k}{7 !}[( & \left.720+1764 k+18564 k^{2}+41685 k^{3}+49665 k^{4}+44751 k^{5}+20511 k^{6}\right) \\
& \left.+210\left(60+286 k+1343 k^{2}+1238 k^{3}+157 k^{4}\right) \beta^{2}-1260\left(95+68 k-36 k^{2}\right) \beta^{4}\right] .
\end{aligned}
$$

Since the coefficient of $\beta^{4}$ is negative, the estimate (8) implies

$$
\begin{gathered}
\left|B_{13}\right| \leqq \frac{k}{7 !}\left[\left(720+1764 k+18564 k^{2}+41685 k^{3}+169365 k^{4}+130431 k^{5}-24849 k^{6}\right)\right. \\
\left.+210\left(60+286 k+203 k^{2}+422 k^{3}+589 k^{4}\right) \beta^{2}\right]
\end{gathered}
$$

Finally, since the resulting coefficient of $\beta^{2}$ is positive, we may replace $\beta^{2}$ by $k^{2}$ and rewrite the bound in the form given in the Principal Theorem.

$n=8$. Just as in the previous case we use (7), the bounds for $\left|B_{3}\right|$ and $\left|B_{5}\right|$, and the estimates $\beta \leqq k$ and $\beta^{3} \leqq k \beta^{2}$ to obtain

$$
\begin{aligned}
\left|B_{8}^{(-6)}\right|^{2} & =36 \beta^{2}, \quad\left|B_{8}^{(-5)}\right|^{2}=25\left|b_{2}\right|^{2} \leqq \frac{25}{2}\left(k^{2}-\beta^{2}\right), \\
\left|B_{8}^{(-4)}\right|^{2} & \leqq\left[4\left|B_{3}\right|+10 \beta^{2}\right]^{2} \leqq\left[2 k(k+1)+10 \beta^{2}\right]^{2}, \\
\left|B_{8}^{(-3)}\right|^{3} & =\left|3 b_{4}+21 b_{1} b_{2}\right|^{2} \leqq\left(\frac{9}{4}+\frac{441}{2} \beta^{2}\right)\left(k^{2}-\beta^{2}\right), \\
\left|B_{8}^{(-2)}\right|^{2} & \leqq\left[2\left|B_{5}\right|+6\left|B_{3}\right| \beta+3\left|b_{2}\right|^{2}+4 \beta^{3}\right]^{2} \\
& \leqq\left(4\left|B_{5}\right|^{2}+24 k\left|B_{3}\right|\left|B_{5}\right|\right)+\left(36\left|B_{3}\right|^{2}+16 k\left|B_{5}\right|\right) \beta^{2}+48\left|B_{3}\right| \beta^{4}
\end{aligned}
$$




$$
\begin{aligned}
& +16 \beta^{6}+\left(6\left|B_{5}\right|+18 k\left|B_{3}\right|+12 k \beta^{2}\right)\left(k^{2}-\beta^{2}\right)+\frac{9}{4}\left(k^{2}-\beta^{2}\right)^{2} \\
\leqq & \frac{1}{36}\left(16 k^{2}+264 k^{3}+1021 k^{4}+1464 k^{5}+700 k^{6}\right) \\
& +\frac{1}{6}\left(-12 k-13 k^{2}+132 k^{3}+166 k^{4}\right) \beta^{2}+\frac{1}{4}\left(9+48 k+96 k^{2}\right) \beta^{4}+16 \beta^{6}, \\
\left|B_{8}^{(-1)}\right|^{2}= & \left|b_{6}+7 b_{1} b_{4}+\left(-7 B_{3}+14 b_{1}^{2}\right) b_{2}\right|^{2} \\
\leqq & {\left[\frac{1}{6}+\frac{49}{4} \beta^{2}+\frac{1}{2}\left(\frac{7}{2} k(k+1)+14 \beta^{2}\right)^{2}\right]\left[k^{2}-\beta^{2}\right] . }
\end{aligned}
$$

Substitution of these bounds into (6) leads to

$$
\begin{aligned}
\left|B_{15}\right| \leqq \frac{k}{8 !}[( & 5040+13068 k+200172 k^{2}+573489 k^{3}+1359120 k^{4}+2089122 k^{5} \\
& \left.+1516788 k^{6}+398721 k^{7}\right)+168\left(620+3928 k+27145 k^{2}+45230 k^{3}\right. \\
+ & \left.28025 k^{4}+3732 k^{5}\right) \beta^{2}-1680\left(1431+2551 k-1326 k^{2}-388 k^{3}\right) \beta^{4} \\
& \left.-80640(45-4 k) \beta^{6}\right] .
\end{aligned}
$$

Since the coefficients of $\beta^{4}$ and $\beta^{6}$ are negative, we may use the estimates (8) and

to obtain

$$
-\beta^{6} \leqq\left(k^{4}-2 k^{2} \beta^{2}\right) \beta^{2} \leqq k^{4} \beta^{2}+2 k^{2}\left(k^{4}-2 k^{2} \beta^{2}\right)=2 k^{6}-3 k^{4} \beta^{2}
$$

$$
\begin{aligned}
\left|B_{15}\right| \leqq \frac{k}{8 !}[ & \left(5040+13068 k+200172 k^{2}+573489 k^{3}+3763200 k^{4}+6374802 k^{5}\right. \\
& \left.+6546708 k^{6}-898239 k^{7}\right)+168\left(620+3928 k-1475 k^{2}-5790 k^{3}\right. \\
& \left.\left.-10255 k^{4}+17252 k^{5}\right) \beta^{2}\right] .
\end{aligned}
$$

One easily shows that the coefficient of $168 \beta^{2}$ is positive. For example, if we denote this polynomial by $p(k)$ and if $0 \leqq k \leqq 1$, then

$$
(1+k) p(k)=k^{2}\left(62-131 k^{2}\right)^{2}+k\left(44-83 k^{2}\right)^{2}+1391 k(1-k)+q(k)>0
$$

where $q(k)=620+1221 k+39 k^{3}+199 k^{4}+108 k^{5}+91 k^{6}>0$. Cosnequently, we may replace $\beta^{2}$ by $k^{2}$ in (9) and rewrite the resulting bound in the form given in the Principal Theorem.

In each case we used the estimate $\beta \leqq k$. Therefore equality can occur only if $\left|b_{1}\right|=k$. For $k=1$ this occurs only for the functions indicated in the Corollary to the Principal Theorem. 


\section{REFERENCES}

[1] Y. Kubota, "Coefficients of meromorphic univalent functions," Ködai Math. Sem. Rep. 28 (1977), 253-261.

[2] R. KuHNAU, "Wertannahmeprobleme bei quasikonformen Abbildungen mit ortsabhängiger Dilatationsbeschränkung," Math. Nachr. 40 (1969), 1-11.

[3] R. KÜHNAU, "Verzerrungssätze und Koeffizientenbedingungen von Grunskyschen Typ für quasikonforme Abbildungen," Math. Nachr. 48 (1971), 77-105.

[4] O. LEHTO, "Schlicht functions with a quasiconformal extension," Ann. Acad. Sci. Fenn. Ser. Al $\# 500$ (1971), 10 pp.

[5] O. LEHTo, "Quasiconformal mappings and singular integrals," Symposia Mathematica, Instituto Nazionale di Alta Mathematica 18 (1976), 429-453.

[6] G. Schober, "Univalent functions-selected topics," Lecture Notes in Math. 478 (1975), Springer-Verlag, $200 \mathrm{pp}$.

[7] G. Schober, "Coefficients of inverses of meromorphic univalent functions," Proc. Amer. Math. Soc. 67 (1977), 111-116.

[8] G. SPRINGER, "The coefficient problem for schlicht mappings of the exterior of the unit circle," Trans. Amer. Math. Soc. 70 (1951), 421-450.

INDIANA UNIVERSITY

BLOOMINGTON, INDIANA

U.S.A. 